\title{
Vibratory Stimulus Reduces In Vitro Biofilm Formation On Tracheoesophageal Voice Prostheses $\$$
}

\author{
Todd J Wannemuehler, $\mathrm{MD}^{1 *}$, Brian C Lobo, $\mathrm{MD}^{1 *}$, Jeffrey D Johnson, MD ${ }^{1,2}$, \\ Christopher R Deig, $\mathrm{BA}^{1}$, Jonathan Y Ting, $\mathrm{MD}^{1}$, Richard L Gregory, $\mathrm{PhD}^{3}$
}

${ }^{1}$ Department of Otolaryngology - Head \& Neck Surgery, Indiana University School of Medicine, Indianapolis, IN, USA

${ }^{2}$ Department of Otorhinolaryngology - Head \& Neck Surgery, University of Texas Medical School at Houston, Houston, TX, USA

${ }^{3}$ Department of Oral Biology, Indiana University School of Dentistry, Indianapolis, IN, USA

${ }^{\S}$ Received Triological Society Middle Section John R. Lindsay, MD, Resident Research Award

${ }^{\S}$ Poster presentation at Triological Society Combined Sections Meeting January 22-24, 2016

*These two authors contributed equally to this work.

${ }^{\sharp}$ This author possesses patent rights for the proprietary hardware utilized in this experiment.

The authors have no other financial relationships or conflicts of interest to disclose.

Running Title: Direct Vibration Reduces Biofilm Formation on TEPs

Word Count: 2,988

Keywords: Tracheoesophageal, Prosthesis, TEP, Vibration, Biofilm, Voice

Level of Evidence: NA (Basic Science Research)

Please send all correspondence to:

Todd J Wannemuehler, MD

Department of Otolaryngology - Head \& Neck Surgery

Indiana University School of Medicine

1120 West Michigan Street, Gatch Hall, Suite 200

Indianapolis, IN 46202

Email: tjwannem@iupui.edu Phone: 317-278-1258

This is the author's manuscript of the article published in final edited form as: 
Objective: Demonstrate that biofilm formation will be reduced on tracheoesophageal prostheses when vibratory stimulus is applied compared to controls receiving no vibratory stimulus in a dynamic in vitro model of biofilm accumulation simulating the interface across the tracheoesophageal puncture site.

Study Design: Prospective, Randomized, Controlled, Crossover in University Laboratory Methods: Ex vivo tracheoesophageal prostheses were obtained from University-affiliated speech language pathologists. Prostheses demonstrating physical integrity and an absence of gross biofilm accumulation were utilized. 16 prostheses were cleansed and sterilized prior to random placement by length in two modified Robbins devices arranged in parallel. Each device was seeded with a polymicrobial oral flora on day 1 and received basal artificial salivary flow continuously with three growth medium meals daily. One device was randomly selected for vibratory stimulus and 2 minutes of vibration was applied to each prosthesis before and after meals for 5 days. The prostheses were explanted, sonicated, and the biofilm cultured for enumeration. This process was repeated after study arm crossover.

Results: Tracheoesophageal prostheses in the dynamic model receiving vibratory stimulus demonstrated reduced gross biofilm accumulation and a significant biofilm colony forming unit per milliliter reduction of 5.56 -fold compared to non-vibratory controls $(\mathrm{p}<0.001)$. Significant reductions were observed within length subgroups.

Conclusion: Application of vibratory stimulus around meal times significantly reduces biofilm accumulation on tracheoesophageal prostheses in a dynamic in vitro model. Further research using this vibratory stimulus method in vivo will be required to determine if reduced biofilm accumulation correlates with longer device lifespan. 


\section{Introduction}

Alaryngeal speech has challenged both patients and their surgeons from the time of the first laryngectomy in 1873 when Theodor Billroth's colleague, Gussenbauer, designed a double cannula speech prosthesis for the first laryngectomee. ${ }^{1}$ Nearly a century later, Mozolewski described the first tracheoesophageal voice prosthesis (TEP), and in 1980, Blom and Singer introduced the duckbill prosthesis which in turn lead to the myriad indwelling and nonindwelling devices available today. ${ }^{2}$ Unfortunately, with what has been described as the most "successful mode of restoring communication after total laryngectomy", 3 also has arisen significant frustration with device failure due in large part to the effects of biofilm accumulation including valve fluid leakage, structural deterioration, and high airflow resistance. ${ }^{4-5}$

Biofilm represents the polymicrobial adherence and replication of various bacterial and fungal species on organic and inorganic surfaces, especially implantable biomedical devices such as the TEP. ${ }^{6}$ Biofilms, mainly those of endogenous oral Candida species, have been attributed to these TEP failures, but the positively synergistic coadherence by bacterial species such as Rothia dentocariosa and Staphylococcus aureus in the biofilm development is also well documented. ${ }^{3,6-}$

${ }^{10}$ Biomaterial studies have actually demonstrated ingrowth of fungal elements into the substance of the silicone prosthesis with subsequent device deterioration and failure. ${ }^{11,12}$ Mean device lifetime averages between 3 to 5 months for most patients before biofilm accumulation results in device malfunction, but some patients require replacement as often as every 3 to 4 weeks. ${ }^{13}$ Device failure is problematic with respect to both patient dissatisfaction and the cost of replacement - including the cost of the device production, the visit to the speech language pathologist, travel costs, and loss of work and personal time.

Wannemuehler, 3 
Attempts to decrease biofilm formation include increased dietary dairy products, enzymes, biosurfactants, and chemical modification of the devices with varying degrees of success. ${ }^{6-10,14,15}$ Most efforts have been aimed at disrupting the initial stages of biofilm formation wherein bacteria and fungi first attach by weak forces to proteinaceous material before more permanent attachment and ingrowth of organisms into the TEP silicone. ${ }^{6}$ Other have utilized biocidal-impregnated silicone materials which secrete low doses of antibiotics in an attempt to curtail bacterial and fungal growth in the forming biofilm rather than prevent initial adherence. ${ }^{6,7}$

An as yet unexplored avenue for reducing biofilm formation and accumulation in TEPs is the application of direct mechanical vibration. Multiple reports detail the use of mechanical and acoustical vibration to diminish biofilm accumulation in the oral cavity. ${ }^{16-18}$ Further studies have demonstrated that even without direct contact, bacterial viability and adherence can be decreased with the application of vibration. ${ }^{19}$ The ubiquity and benignity of electric sonicating toothbrushes offers further evidence that patients are familiar with the technology and its use in preventing biofilm associated dental caries. ${ }^{18-19}$ The promise of mechanical vibration application lies in the possibility of both reducing initial adherence as well as continually disrupting the growth of microorganisms forming biofilm.

With this in mind, the purpose of this present study is to elucidate whether a vibratory stimulus applied directly to TEPs will reduce the amount of biofilm accumulation. An initial stage of testing utilizing a static model of biofilm formation demonstrated gross proof of concept. In this, a polymicrobial suspension including Candida albicans, Candida tropicalis, Streptococcus salivarius, Rothia dentocariosa, Staphylococcus aureus, and Staphylococcus epidermidis was applied to TEPs partially submerged in growth medium in sterile well culture plates. Vibratory stimulus was applied to the treatment group during the 4 day growth course. 
Cultures from TEPs demonstrated some reduction in colony forming unit (CFU) per milliliter $(\mathrm{mL})$ in those treated with the vibratory stimulus compared to controls. The hypothesis of this current research, however, moves one step further in positing that mechanical vibration applied directly to the TEP will reduce the accumulation of biofilm on TEPs in a dynamic microcosmic system utilizing a modified Robbins device (MRD) which emulates the tracheoesophageal puncture site interface.

\section{Materials and Methods}

\section{Tracheoesophageal Voice Prostheses Preparation}

Expired and ex vivo Atos Medical ${ }^{\circledR}$ (West Allis, Wisconsin) Provox ${ }^{\circledR 2}$ low-resistance, indwelling silicone voice prostheses of 22.5 French (F) internal diameter and shaft lengths of 6 millimeter $(\mathrm{mm}), 8 \mathrm{~mm}$, and $10 \mathrm{~mm}$ were utilized. TEPs were provided by University-affiliated speech language pathologists. Grossly contaminated TEPs with visibly deteriorated silicone unamenable to cleaning and decontamination were excluded. The included TEPs were individually scrubbed, sonicated for 15 seconds (s), rinsed with deionized water, disinfected for 30 minutes $(\mathrm{min})$ in $70 \%$ ethanol, and dried in aseptic conditions prior to loading into the MRDs. TEPs were stratified by length and then equal quantities of the length groups were randomly assigned to placement in either the vibration arm MRD or the control arm MRD.

\section{Modified Robbins Device Setup}

The MRD is a common system used in microbiological studies on biofilm formation. ${ }^{20}$ It involves a closed system of tubing with an influent source of liquid nutrition moved by a peristaltic pump through a unidirectional device chamber which has effluent tubing to waste. For this research, a special modification of the Robbins device was employed which particularly 
emulated the tracheoesophageal puncture site interface. The device chamber itself was manufactured from an autoclavable polymer. A silicone sheet representing the tracheoesophageal party wall was punctured and the TEPs were loaded with the esophageal TEP face positioned inside the device for exposure to systemic flow while the tracheal TEP face was exposed to ambient air (Figure 2). This arrangement allows for exposure of the TEP to basal salivary flow as well as nutrient rich meals similar to the microbiological milieu which would exist in vivo in a laryngectomee.

The experimental setup involved three separate influent sources containing 1) normal saline (representing saliva), 2) a 1:1 mixture growth medium (GM) of Brain Heart Infusion (BHI) and Yeast Extract-Peptone-Dextrose (YPD) broths, and 3) a smaller 1:1 mixture GM of $\mathrm{BHI}$ and YPD broths to be inoculated (after autoclaving) with a polymicrobial culture of $C$. albicans, C. tropicalis, S. salivarius, R. dentocariosa, S. aureus, and S. epidermidis. Autoclavable tubing from the three fluid sources were Y-connected together before splitting into separate parallel tubing through 2 cassettes in an Ismatec ${ }^{\circledR}$ Reglo Digital 2 channel peristaltic pump (IDEX Corp, Wertheim, Germany). From the pump, the parallel tubing connected to the influent spigots of two MRDs each with effluent spigot tubing dumping into an effluent beaker (Figure 3). The parallel MRDs were utilized in an effort to create a microcosmic dynamic tracheoesophageal puncture site interface while reducing intergroup variability as each MRD received the same influent fluids and flow rates from the same source containers. The entire system of flasks, tubing, and MRDs was autoclaved to ensure internal sterility prior to starting the experimental run in an aerobic $5 \% \mathrm{CO}_{2}$ incubator set at $37^{\circ} \mathrm{C}$.

Wannemuehler, 6 


\section{Inoculation, Simulated “Meal” Feeding Cycles, and Biofilm Formation}

Twelve hours prior to placement of the experimental system in the aerobic incubator, individual broth cultures of C. albicans, C. tropicalis, S. salivarius, R. dentocariosa, S. aureus, and S. epidermidis were inoculated and incubated aerobically with $5 \% \mathrm{CO}_{2}$ at $37^{\circ} \mathrm{C}$. On the first morning of the experiment, $1 \mathrm{~mL}$ of each of these culture broths were aseptically added to the smaller GM container to provide polymicrobial inoculation of the MRDs. During the first day of the experimental run, the inoculated GM was pumped through the system at a rate of $1 \mathrm{~mL} / \mathrm{min}$ for 30 min on 3 occasions to simulate 3 meals evenly spaced throughout the day. A normal saline flow rate of $0.5 \mathrm{~mL} / \mathrm{min}$ between meals simulates wakeful unstimulated salivary flow rates whereas a rate of $0.1 \mathrm{~mL} / \mathrm{min}$ simulates resting salivary flow rates between evening and morning. ${ }^{21}$ On experimental days 2 through 5, the sterile GM source was utilized to simulate meals as the system was adequately inoculated. The entire experimental setup was maintained in the aerobic incubator at $37^{\circ} \mathrm{C}$ while biofilm formed over 5 days.

\section{Mechanical Vibration Application}

One MRD was randomly selected to have its TEPs undergo mechanical vibration. Vibration was applied to TEPs mounted in the treatment group MRD by a motor oscillating at approximately $260 \mathrm{hz}$ for $2 \mathrm{~min}$ duration before and after each "meal" to simulate when vibration would reasonably be performed by a patient. This vibration frequency and duration has proven safe for long term intraoral use by electric toothbrush manufacturers.

\section{Biofilm Culturing and Colony Counting}

At the conclusion of 5 days, the TEPs were aseptically removed individually from the MRDs, unbound bacteria were gently rinsed away with sterile normal saline, and the TEPs were placed in individual $50 \mathrm{~mL}$ sterile conical centrifugation tubes containing $5 \mathrm{~mL}$ of sterile saline. 
Each explanted TEP was then vortexed for $15 \mathrm{~s}$, sonicated for $15 \mathrm{~s}$, and again vortexed for $15 \mathrm{~s}$ to release the biofilm microorganisms into suspension. For each TEP, the $5 \mathrm{~mL}$ undiluted biofilm suspensions were diluted 1:200 and 1:1,000 prior to duplicate culturing of each dilution onto 2 blood agar plates with a spiral plating apparatus. The culture plates were left to dry at room temperature for 15 min prior to aerobic incubation with $5 \% \mathrm{CO}_{2}$ for 36 hours. The ProtoCOL® 1 automated colony counting system (Synbiosis, Frederick, Maryland) was utilized to determine CFU/mL for each plate. Three measurements each for the 2 blood agar plates for the 1:1,000 dilution were averaged as they demonstrated appropriately countable colony density. The 1:200 plates were not counted because of the high density of the colonies.

\section{Experimental Crossover and Duplication}

At the conclusion of the 5 day experimental run, the entire MRD system (including the TEPs) was disassembled and decontaminated with 70\% ethanol prior to scrubbing, rinsing with deionized water, and reassembly. New GM and normal saline were prepared and the TEPs were replaced in fresh silicone sheets within the MRD chambers. The experimental system was again run with the previously described inoculation methods, flow rates, and time frames. However, during the duplication run the TEPs underwent experimental arm crossover and the previously non-vibration group had mechanical vibration applied as described above. Subsequently, the TEPs underwent explantation, culturing, and colony counting with the same methods as the first run.

\section{Statistical Considerations}

The coefficient of variation in this experiment was anticipated to be approximately 0.6 based on a prior study. ${ }^{7}$ With a total sample size of 12 per group, the study was anticipated to have $80 \%$ power to detect a 2 times difference in $\mathrm{CFU} / \mathrm{mL}$ between groups, at an alpha of $5 \%$ 
significance level. Because of the plan for subgroup analysis by length, a total of 16 TEPs were utilized in each run ( 8 per MRD). Each MRD was populated by four $6 \mathrm{~mm}$ length TEPs, three $8 \mathrm{~mm}$ TEPs, and a single $10 \mathrm{~mm}$ TEP. Grouping of TEPs into the treatment MRD or control MRD was randomized by length. A paired-samples t-test was utilized for cumulative treatment arm analysis and to compare vibratory versus non-vibratory groups at different TEP lengths. Insufficient blood agar resulted in an inability to culture and measure biofilm formation in the non-vibratory group's 10mm TEP sample. Consequently, the paired $10 \mathrm{~mm}$ vibratory TEP sample was excluded from paired analysis. An independent-samples two-tailed t-test was used for intra-run analysis between vibratory and non-vibratory groups and for inter-run analysis (vibratory versus vibratory; non-vibratory versus non-vibratory). One-way ANOVA was used to compare the effect of TEP length on $\mathrm{CFU} / \mathrm{mL}$ cultured. A natural log transformation was performed to allow for parametric analysis. All variance within paired analysis was expressed as standard error of the mean (SEM) of the differences between paired samples. The variance within one-way ANOVA and independent-samples t-tests was expressed as SEM. Statistical analysis was accomplished using SPSS ${ }^{\circledR} 22$ (IBM Corp).

\section{Results}

When comparing all vibratory TEPs to non-vibratory TEPs for both experimental runs, there was a statistically significant 5.56-fold reduction in mean CFU/mL (See Table 1, Figure 4). When comparing the treatment arm to the control arm for each run individually, significant reductions in $\mathrm{CFU} / \mathrm{mL}$ were also observed. The first run showed a statistically significant 3.6fold reduction between the mean $\mathrm{CFU} / \mathrm{mL}\left(2.0 \times 10^{6} \pm 0.21 \times 10^{6} \mathrm{CFU} / \mathrm{mL}\right.$ (non-vibratory group) versus $0.55 \times 10^{6} \pm 0.10 \times 10^{6} \mathrm{CFU} / \mathrm{mL}$ (vibratory group); $\mathrm{t}(13)=-6.39, p=0.000024$ ) 
Within the second run there was a significant 9.4-fold reduction between the non-vibratory and vibratory mean $\mathrm{CFU} / \mathrm{mL}$ with analysis following a natural log transformation: $14.4 \pm 0.28$ $\ln (\mathrm{CFU} / \mathrm{mL})$ versus $12.2 \pm 0.25 \ln (\mathrm{CFU} / \mathrm{mL}) ;(\mathrm{t}(14)=5.70, \mathrm{p}=0.000055)$.

Since there was a wide range in reduction of biofilm between the first and second runs (3.6-fold versus 9.4-fold, respectively), post-hoc subgroup analyses were performed. The first run's non-vibratory group mean $\mathrm{CFU} / \mathrm{mL}$ was compared to the second run's non-vibratory group, and no significant difference was observed following a natural log transformation (14.4 \pm $0.12 \ln (\mathrm{CFU} / \mathrm{mL})$ versus $14.4 \pm 0.28 \ln (\mathrm{CFU} / \mathrm{mL}) ; \mathrm{t}(13)=0.26, p=0.80)$. The vibratory group mean $\mathrm{CFU} / \mathrm{mL}$ between the first and second runs were similarly analyzed, and in this case a significant difference was observed $\left(0.55 \times 10^{6} \pm 0.010 \times 10^{6} \mathrm{CFU} / \mathrm{mL}\right.$ versus $0.25 \times 10^{6} \pm 0.062$ $\left.\mathrm{x} 10^{6} \mathrm{CFU} / \mathrm{mL} ; \mathrm{t}(14)=2.60, p=0.021\right)$.

For all TEP length subgroups analyzed, significant reductions in mean $\mathrm{CFU} / \mathrm{mL}$ were observed after vibratory treatment with a trend toward higher $\mathrm{CFU} / \mathrm{mL}$ reduction in larger TEP lengths (Table 1, Figure 5). One-way ANOVA comparing mean $\mathrm{CFU} / \mathrm{mL}$ demonstrated no significant difference between $6 \mathrm{~mm}, 8 \mathrm{~mm}$, or $10 \mathrm{~mm}$ length groups in both the vibratory treatment group $\left(F_{2,13}=1.55, p=0.25\right)$ and non-vibratory group $\left(F_{2,12}=3.84, \mathrm{p}=0.052\right)$.

\section{Discussion}

In this present study which utilized a dynamic in vitro model of the tracheoesophageal puncture site interface, the application of vibration to TEPs resulted in an overall statistically significant 5.56-fold reduction in biofilm accumulation. Moreover, the statistically significant reduction in $\mathrm{CFU} / \mathrm{mL}$ was observed in $6 \mathrm{~mm}$ and $8 \mathrm{~mm}$ length subgroup analyses as well (Table 1). Interestingly, as TEP length increased, the proportional reduction in $\mathrm{CFU} / \mathrm{mL}$ also increased; 
a 5.0 fold reduction for $6 \mathrm{~mm}$ TEPs and a 5.6 fold reduction for $8 \mathrm{~mm}$ TEPs. This may be related to the potential stasis of GM and bacteria within the longer TEP shaft by capillary forces. Prolonged stasis of sonicated biofilm elements may allow more rapid re-accumulation of biofilm in these larger TEPs. Analysis revealed that within both the vibratory and non-vibratory groups, length did not significantly affect $\mathrm{CFU} / \mathrm{mL}$ cultured. Therefore, mechanical vibration appears to have the strongest effect among the potential variables influencing biofilm formation in this study.

One of the strengths of this present study was the parallel arrangement of MRDs within a run and the crossover of treatment arms between runs. These measures confirmed that the reduction in $\mathrm{CFU} / \mathrm{mL}$ was not likely related to local growing condition variability nor intrinsic susceptibility for biofilm formation by a TEP subset. Despite endeavoring to reduce variability, it should be noted that between the first and second run, there existed a significant difference in $\mathrm{CFU} / \mathrm{mL}$ between each run's vibratory group while finding no difference between each run's non-vibratory group. One explanation for this is found in the intrinsically stochastic nature of polymicrobial cultures. There is a possibility that different bacterial species' success was variable in early TEP adherence, and that some of these species are more resistant to the biofilm reducing effect of vibration. All culture plates demonstrated polymorphic colony types consistent with polymicrobial growth, but speciation techniques were not utilized. Despite the difference between the two runs' vibratory groups, however, each vibratory group was significantly different compared to its non-vibratory counterpart within their respective runs.

A potential limitation of the present study was the relative brevity of each experimental run (5 days) compared to the minimum expected longevity of TEP device life of 4-8 weeks. However, a previous proprietary study by our lab revealed appreciable biofilm accumulation by 
3 to 4 days and this study was focused on demonstrating biofilm reduction rather than on analyzing device longevity. The biofilm CFU $/ \mathrm{mL}$ on the order of $10^{6}$ observed in our study was consistent with that of previous studies, thus we posit our experimental duration was appropriate..$^{7,19}$

\section{Conclusion}

Vibratory stimulus application similar to that which has been used in the oral health industry to prevent dental caries for years has been shown in this study to significantly reduce biofilm formation on TEPs in vitro. Further in vitro longitudinal studies are needed to demonstrate whether this reduction in biofilm accumulation is maintained over the course of weeks. Research in the clinical setting will be necessary to demonstrate that this in vitro reduction in biofilm correlates with longer device lifespan in vivo and to determine the optimal frequency and duration of vibration application to maximize patient compliance. This novel approach to TEP maintenance demonstrates promise in reducing the frustration of TEP failure and the cost of frequent TEP replacement by many laryngectomees. 


\section{Author Contributions}

Todd J Wannemuehler, study design, data collection, data analysis and interpretation, article drafting, article revision; Brian C Lobo, study design, data collection, article drafting, article revision; Jeffrey D Johnson, study design, data collection, article revision; Christopher R Deig, data analysis and interpretation, article drafting, article revision; Jonathan Y Ting, study design and article revision. Richard L Gregory, study design, data analysis and interpretation, article revision.

\section{Disclosures}

Competing interests: The second author possesses patent rights for the proprietary MRD hardware utilized in this experiment.

Sponsorships: None.

Funding source: Indiana University School of Medicine Department of Otolaryngology - Head \& Neck Surgery provided basic funds for culture media. No role in study.

\section{Acknowledgments}

Stacey L. Halum, MD, who participated in initial study conceptualization and design.

Ruijie Huang, DDS, PhD, who participated in study design. 


\section{References}

1. Bień S, Rinaldo A, Silver CE, et al. History of voice rehabilitation following laryngectomy. Laryngoscope. 2008; 118(3):453-8. doi: 10.1097/MLG.0b013e31815db4a2.

2. Mozolewski E. Surgical rehabilitation of voice and speech following laryngectomy. Otolaryngol Pol. 1972; 26(6): 653-661.

3. Op de Coul BM, Hilgers FJ, Balm AJ, Tan IB, van den Hoogen FJ, van Tinteren H. A decade of postlaryngectomy vocal rehabilitation in 318 patients: a single institution's experience with consistent application of Provox indwelling voice prostheses. Arch Otolaryngol Head Neck Surg. 2000; 126(11):1320-1328.

4. Hancock KL, Lawson NR, Ward EC. Device life of the Provox Vega voice prosthesis. Eur Arch Otorhinolaryngol. 2013; 270(4):1447-53. doi: 10.1007/s00405-012-2154-9.

5. Laccourreye O, Menard M, Crevier-Buchman L, Couloigner V, Brasnu D. In situ lifetime, causes for replacement, and complications of the Provox voice prosthesis. Laryngoscope. 1997; 107(4):527-530.

6. Rodrigues LR, Banat IM, Teixeira JA, Oliveira R. Strategies for prevention of microbial biofilm formation on silicone rubber voice prostheses. J Biomed Mater Res B Appl Biomater. 2007; 81(2):358-70.

7. Oosterhof JJ, Buijssen KJ, Busscher HJ, van der Laan BF, van der Mei HC. Effects of quaternary ammonium silane coatings on mixed fungal and bacterial biofilms on tracheoesophageal shunt prostheses. Appl Environ Microbiol. 2006; 72(5):3673-3677. doi: 10.1128/aem. 
8. Free RH, van der Mei HC, Elving GJ, Van Weissenbruch R, Albers FW, Busscher HJ. Influence of the Provox flush, blowing, and imitated coughing on voice prosthetic biofilms in vitro. Acta Otolaryngol. 2003; 123(4):547-55.

9. Elving GJ, van der Mei HC, Busscher HJ, et al. Antimicrobial activity of synthetic salivary peptides against voice prosthetic microorganisms. Laryngoscope. 2000; 110(2 Pt 1):321324.

10. Schwandt LQ, van Weissenbruch R, Stokroos I, van der Mei HC, Busscher HJ, Albers FW. Prevention of biofilm formation by dairy products and $\mathrm{N}$-acetylcysteine on voice prostheses in an artificial throat. Acta Otolaryngol. 2004; 124(6):726-731.

11. Neu T, van der Mei HC, Busscher HJ, Dijk F, Verkerke G. Biodeterioration of medicalgrade silicone rubber used for voice prostheses: a SEM study. Biomaterials. 1993; 14(6):459-64. 12. van der Mei HC, van de Belt-Gritter B, Dijk F, Busscher HJ. Initial deterioration of silicone rubber by Candida albicans and Candida tropicalis strains isolated from voice prostheses. Cells Mater. 1996; 6:157-63.

13. Tićac B, Tićac R, Rukavina T, et al. Microbial colonization of tracheoesophageal voice prosthesis (Provox2) following total laryngectomy. Eur Arch Otorhinolaryngol. 2010; 267:1579-1586.

14. Rodrigues LR, Banat IM, Teixeira JA, Oliveira R. Biosurfactants: potential applications in medicine. J Antimicrob Chemother. 2006; 57(4):609-18.

15. Smith A, Buchinsky FJ, Post JC. Eradicating chronic ear, nose, and throat infections: a systematically conducted literature review of advances in biofilm treatment. Otolaryngol Head Neck Surg. 2011; 144(3):338-347. 
16. McInnes C, Engel D, Monicia BJ, Martin RW. Reduction in adherence of Actinomyces viscosus after exposure to low-frequency acoustic energy. Oral Microbiol Immunol. 1992; $7: 171-176$.

17. McInnes C, Engel D, Martin RW. Fimbria damage and removal of adherent bacteria after exposure to acoustic energy. Oral Microbiol Immunol. 1993; 8(5):277-282.

18. Wuyan CD, Anderson R, Keller JD, Stanford C. Removal of adherent oral bacteria from titanium surface by Sonicare. J Dent Res. 1994; 73:331.

19. Hope CK, Wilson M. Effects of dynamic fluid activity from an electric toothbrush on in vitro oral biofilms. J Clin Periodontol. 2003; 30(7):624-629.

20. Kharazmi A, Giwercman B, Høiby N. Robbins device in biofilm research. Methods Enzymol. 1999; 310:207-15.

21. Coulthard P, Horner K, Sloan P, Theaker E. Oral and Maxillofacial Surgery, Radiology, Pathology and Oral Medicine. $2^{\text {nd }}$ ed. Edinburgh: Churchill Livingstone/Elsevier. pp. 210, 212213. 2008. 


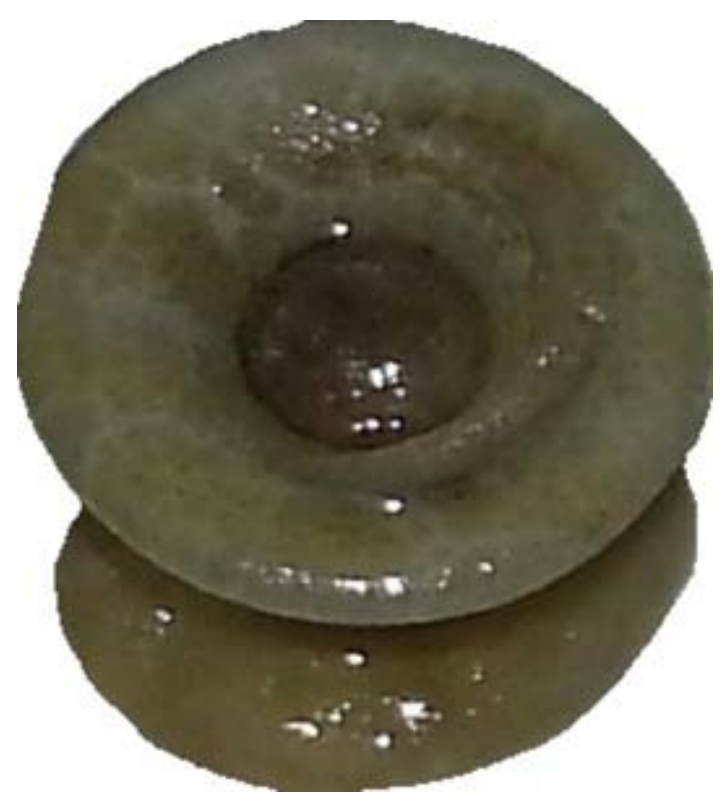

Figure 1. Explanted non-functioning TEP with gross biofilm accumulation.

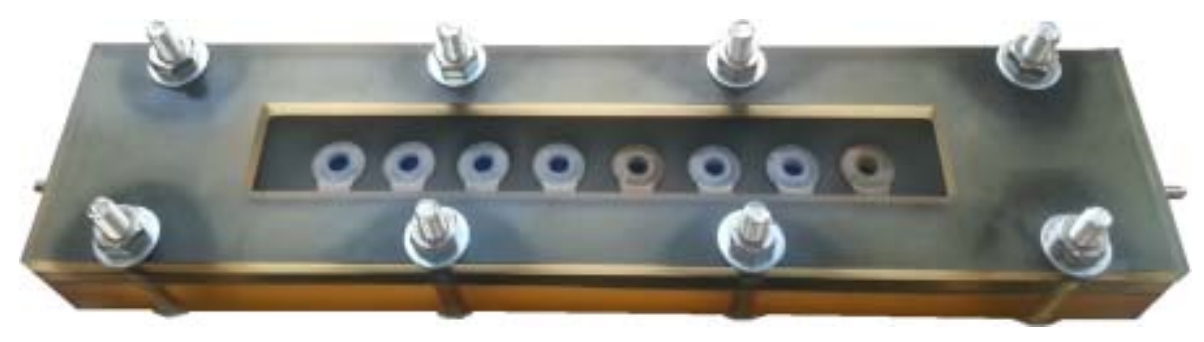

Figure 2. Proprietary Modified Robbins Device with TEPs.

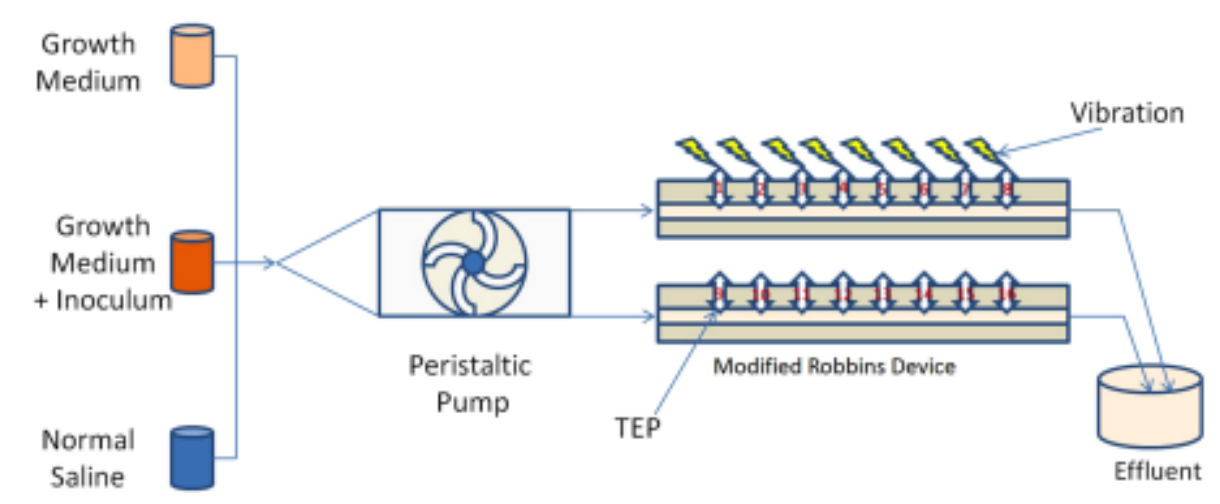

Figure 3. Experimental Design Diagram.

Wannemuehler, 17 


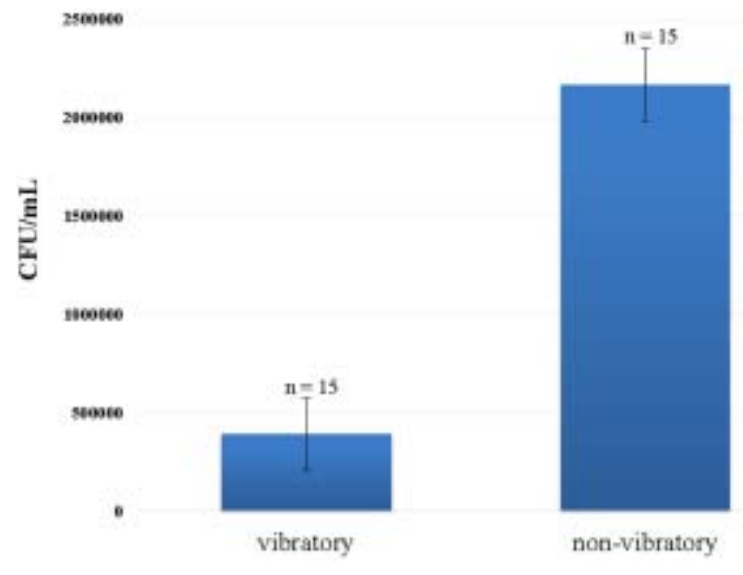

Figure 4. Comparison of paired vibratory and non-vibratory groups with all TEP lengths and runs included (excluding unpaired vibratory $10 \mathrm{~mm}$ TEP in first run). The vibratory group's mean $\mathrm{CFU} / \mathrm{mL}\left(0.39 \times 10^{6} \pm 0.072 \times 10^{6}\right)$ is significantly different than the non-vibratory group $(2.2 \times$ $\left.10^{6} \pm 0.41 \times 10^{6}\right) ; \mathrm{t}(14), p=0.00025$. Error bars represent SEM of the differences between paired samples.

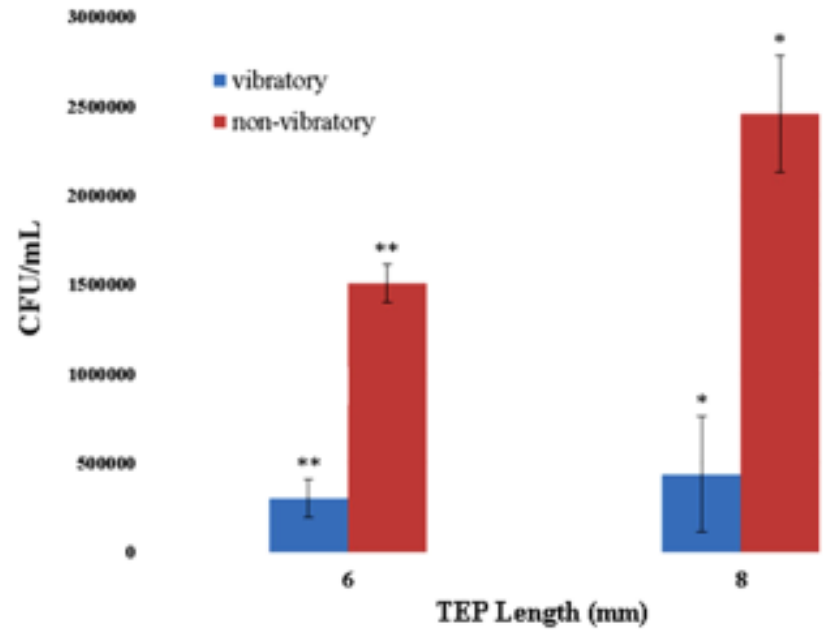

Figure 5. Comparison of TEP length and CFU/mL for paired samples within vibratory and nonvibratory groups. There are statistically significant differences between vibratory and nonvibratory groups with $6 \mathrm{~mm}$ and $8 \mathrm{~mm}$ TEPs. $*=p<0.05 ; * *=p<0.001$ comparing vibratory 
and non-vibratory groups at each TEP length. Error bars represent SEM of the differences between paired samples.

Wannemuehler, 19 
Table 1. Comparison of Mean CFU/mL Formed in Vibratory and Nonvibratory Groups Based on TEP Length.

\begin{tabular}{|c|c|c|c|c|c|}
\hline $\begin{array}{l}\text { TEP } \\
\text { Length } \\
(\mathrm{mm})\end{array}$ & $\begin{array}{l}\text { Vibratory } \\
\text { Group } \\
\text { (CFU } / \mathrm{mL})\end{array}$ & $\begin{array}{l}\text { Nonvibratory } \\
\text { Group (CFU/mL) }\end{array}$ & (n) & $\begin{array}{l}t \\
\text { Value }\end{array}$ & Significance \\
\hline 6 & $\begin{array}{l}0.30 \times 10^{6} \pm 0.11 \times \\
10^{6}\end{array}$ & $1.5 \times 10^{6} \pm 0.11 \times 10^{6}$ & 16 & $\begin{array}{l}t(7)= \\
-5.64\end{array}$ & $0.00078^{b}$ \\
\hline 8 & $\begin{array}{l}0.44 \times 10^{6} \pm 0.33 \times \\
10^{6}\end{array}$ & $2.5 \times 10^{6} \pm 0.33 \times 10^{6}$ & 12 & $\begin{array}{l}\mathrm{t}(5)= \\
-3.10\end{array}$ & $0.027^{\mathrm{b}}$ \\
\hline 10 & $0.79 \times 10^{6}(n=1)$ & $5.7 \times 10^{6}(n=1)$ & 2 & $a$ & $a$ \\
\hline Combined & $\begin{array}{l}0.39 \times 10^{6} \pm 0.18 \times \\
10^{6}\end{array}$ & $2.2 \times 10^{6} \pm 0.18 \times 10^{6}$ & 30 & $\begin{array}{l}t(14)= \\
-4.86\end{array}$ & $0.00025^{b}$ \\
\hline \multicolumn{6}{|c|}{$\begin{array}{l}P \text {-values (Sig.) are derived from paired samples } t \text { tests comparing vibratory and nonvibratory groups } \\
\text { within each row. Standard error of the mean of the differences between paired samples is used to } \\
\text { express variability. }\end{array}$} \\
\hline \multicolumn{6}{|c|}{ a Statistical analysis could not be performed due to insufficient sample size in 10-mm TEP group. } \\
\hline \multicolumn{6}{|c|}{ b Denotes statistical significance at $95 \%$ confidence interval $(P<0.05)$} \\
\hline $\mathrm{CFU}=$ colo & ming unit; TEP = trac & esophageal voice prost & & & \\
\hline
\end{tabular}

